$15^{\text {th }}$ International Conference on

AEROSPACE SCIENCES \& AVIATION TECHNOLOGY,

ASAT - 15 - May 28 - 30, 2013, Email: asat@mtc.edu.eg,

Military Technical College, Kobry Elkobbah, Cairo, Egypt,

Tel: +(202) $24025292-24036138$, Fax: + +(202) 22621908

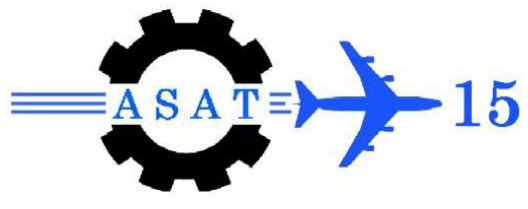

\title{
Factors Affecting Burning Rate of Hydroxy Terminated Polybutadiene Propellant
}

\author{
A. A. E. Osman ${ }^{*}$, M. H. M. Abuuznien ${ }^{\dagger}$, B. K. Abdallah ${ }^{\ddagger}$
}

\begin{abstract}
Burning rate is one of the most important characteristics of rocket propellants and it is affected by many factors including composition, pressure, initial temperature, oxidizer particles size and burning rate modifier. This research aims to study experimentally the effects of combustion chamber pressure, initial temperature and particles size of the oxidizer material on the burning rate of solid composite propellant. Besides, study theoretically the effect of composition and catalyst used. In addition, this research aims to show the relationship between these factors and the burning rate in order to evaluate factor that is more effective on burning rate, so to calculate of burning rate as a function of this factor. Accordingly, this could be used to achieve ballistics characteristics required for rocket motor design and performance.
\end{abstract}

Keywords: Composite propellant, Burning rate.

\section{Nomenclature}

$\begin{array}{ll}\mathrm{AP} & \text { Ammonium Per-chlorate } \\ \mathrm{Al} & \text { Aluminum } \\ \mathrm{a} & \text { Temperature Coefficient in Sant Robert Equation } \\ \mathrm{a}_{0} & \text { Initial Temperature Constant } \\ \mathrm{DOS} & \text { Dioctyl Sebacate } \\ \mathrm{HX}-103 & \text { Antioxidant } \\ \mathrm{MAPO} & \text { Tri-2-methy-1-aziridinyl Phosphine Oxide } \\ \mathrm{n} & \text { Pressure Exponent } \\ \mathrm{T}_{\text {ig }}(\mathrm{K}) & \text { Ignition Temperature } \\ \mathrm{T}_{\text {in }}(\mathrm{K}) & \text { Initial Temperature } \\ \text { TBfe } & \text { Try Butyl Ferrocene }\end{array}$

\section{Introduction}

Several factors affect the rate of burning of propellants. The most important of them are:

\subsection{Chemical Composition}

In the composite propellant, the binder plays an important role in combustion of propellant and affects the value of exponent of burning rate - pressure curve of composite propellant. During the combustion of propellant, the binder can change the flame temperature, decomposition process of $\mathrm{AP}$ as well as gas -phase reaction process, heat balance and structure of combustion surface of propellant. Another main ingredient in composite

Lecturer, Chemical Engineering Dep., Karary University, Sudan.

$\dagger \quad$ Associate Professor, Head of Dep. of Chemical Engineering, Karary University, Sudan.

* Associate Professor, Chemical Engineering Dep., Karary University, Sudan. 
propellant is the oxidizer which represents the main source of oxygen for combustion of binder and metal fuel, it accounts for big percentage in propellant, therefore, the performance, contents, particle size and mean size distribution of oxidizer have great effect on combustion performance and burning rate of propellant. On the other hand, adding metal powder like aluminum in composite propellant can not only improve energy and suppress instable combustion but also influence the burning rate characteristics. The effect of aluminum powder and its particle size on burning rate of propellant is complex, for example in HTPB high burning rate propellant formulation the burning rate of propellant with aluminum powder with particle size of $60 \mu \mathrm{m}$ is $1.9 \%-12.3 \%$ higher than with particle size of $23 \mu \mathrm{m}$, in spite of that, it is reported that the burning rate of HTBP propellant can be doubled if the general aluminum powder has been replaced with $0.5 \mu \mathrm{m}$ super fine aluminum powder. This is as a result that super fine aluminum powder acts as exothermic agent [1].

\subsection{Initial Temperature}

The dependence of burning rate on initial temperature is particularly important for missiles that encounter a wide range of temperatures in the course of their operation [2]. Increase in the initial temperature of a propellant causes an increase in the burning rate. Decrease in the burning rate because of decrease in initial temperature results in decreased pressures and causes difficulty in achieving satisfactory ignition.

\subsection{Pressure}

Pressure here means pressure of confinement or pressure of combustion chamber. Generally, it is believed that the higher is the pressure the higher is the rate of burning of the propellant provided other conditions are constants. When a propellant starts to burn in a chamber the pressure is nearly equal atmospheric pressure and the burning rate is comparatively low. Then the pressure begins to increase due to evolving gases and the increasing of pressure in turn leads to increasing of burning rate according to the following reasons:

a) In most of the oxidation reactions, increasing of pressure increases the extent of reactions and so increases the heat released from the exothermic burning reactions and so increases the burning rate [3]

b) The pressure acts as a factor enforces the hot gaseous products to be close to the burning surface which lead to increasing of the surface temperature and that increases the thermal pyrolysis of the propellant which means increasing of the burning rate $[4,6]$.

c) Due to the all gas phase state equations, increasing of pressure in a constant confined- volume leads to increasing of temperature and accordingly the rate of burning reaction [5].

Vieille and Sarrau proposed the following experimental equation [7]:

$\mathrm{r}=\mathrm{a} \mathrm{P}^{\mathrm{b}}$

where

$\mathrm{r}=$ Burning rate, $\mathrm{P}=$ Pressure, $\mathrm{a}=$ The Initial temperature coefficient.

$\mathrm{a}=\mathrm{a}_{\mathrm{o}} /\left(\mathrm{T}_{\mathrm{ig}}-\mathrm{T}_{\mathrm{in}}\right)$

where

$\mathrm{a}_{\mathrm{o}}=$ constant, $\mathrm{T}_{\mathrm{ig}}=$ ignition temperature, $\mathrm{T}_{\mathrm{in}}=$ initial temperature. 


\section{Particle Size}

Generally, the greater is the surface exposed to combustion, the faster is the burning process, provided other factors such as composition and confinement pressure are the same in all tests. There are three type of particle size for (AP), fine, medium and coarse (see table 1) [7].

Aluminum fuel - in the AP - HTBP composite propellant - particle size has also an influence on the burning rate but that is much less pronounced than that of oxidizer. Besides, its particle size variation has a little effect except with ultra-fine powders, further more ignition and combustion of metals take place in the gas stream [6].

\section{Burning Rate Modifiers}

A burning rate modifier or burning rate catalyst helps to accelerate or decelerate the combustion at the burning surface and increases or decreases the value of the propellant burning rate - pressure exponent. These modifiers are effective because they change the combustion mechanism. Generally, modifiers can be classified into burning rate moderators and burning rate accelerators. Burning rate moderators includes inorganic salts and its mixture, hydroxylamine and organic acid salts. Burning rate accelerators such as ferric oxide and n-butyleferrocene are used to increase the rate of burning [8].

\section{Materials and Methods}

\subsection{Equipment.}

Equipment used in this study to produce different propellant formulae include, preheating oven, AP crusher, particle size analyzer, five liter mixer, viscometer, propellant casting system, curing oven and digital balances. Other equipment were used for testing such as mechanical tester to determine the mechanical properties of the propellant after curing and strand burner that worked under high pressure and low to high temperature to determine burning rate as a function of pressure and then determine the values of temperature coefficient and pressure exponent. Ground static testing station is also used to test ballistic evaluation motor (BEM) to determine the performance and ballistic characteristics of the propellant.

\subsection{Materials}

Table (1) shows different ingredients used to formulate the AP-HTPB Propellant and their functions. Three formulae (F1, F2, and F3) were produced and tested.

\subsection{Method}

The following steps represent the procedure of experimental work to study the main factors affecting burning rate.

a) Specific formulation was prepared with equal AP particle size distribution. This formulation was designated as F1.

b) Above formulation was divided into a block and a small ballistic evaluation motor BEM.

c) Thirteen strands - samples - were made from the block, and six samples were used to determine burning rate, pressure exponent and temperature coefficient. Tests were made at three different initial temperatures $\left(10,20\right.$, and $\left.30^{\circ} \mathrm{C}\right)$ and four different pressures $(4,6,9$, and $11 \mathrm{MPa})$. One strand was used to determine mechanical properties by mechanical tester.

d) Static test was executed by BEM to determine the performance and the ballistic characteristics of propellant F1. 
e) Two other formulations of propellant were produced using different particles size distributions, these formulations are called F2, which represent the maximum amount of fine AP particle size and F3 which represent the maximum amount of coarse AP particle size. These formulations were tested as before.

\section{Results and Analysis}

Applying strand burner test and measuring at different pressures and initial temperatures, burning rates are obtained in the Table (2).

Applying method of standard EBM testing and measuring at different AP particle size distributions, average pressures and burning rates are obtained in the Table (3).

Charts representing the relation between burning rate and pressure at different particle size

distributions and different initial temperatures are obtained (Figures 1 to 3)

Statistical analysis program SPSS was used to find that the relation between burning rate and pressure according to the equation of Sant Rober. Results show that values of temperature coefficient (a) and exponent (n) vary with particle size distribution and temperature as shown in Table (4).

\section{Discussion}

From above results and analysis, the following points can be deduced:

a) For the same AP particle size and initial temperature, the burning rate increases as the pressure increases. This agrees the theory in this area.

b) For each AP particle size distribution and at a fixed pressure, the burning rate increases as the initial temperature increases. But the effect of initial temperature on the burning rate is less than that of pressure and this is because the effect of initial temperature is restricted at the beginning of burning while pressure affects the burning all throughout the burning course.

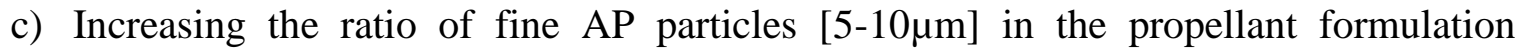
increases the burning rate. This can be attributed to the increase $f$ the surface area on which the burning process occurs as a result of increasing the ratio of fine particles.

d) The value of pressure exponent (n) decreases as initial temperature increases which reduces the effect of pressure on the burning rate and this agrees the theory in this area.

e) The value of temperature coefficient (a) increases as initial temperature increases and this also agrees with theory in this area.

\section{Conclusion}

According to the analysis of results the following points can be concluded:

a) As AP particle size is reduced burning rate increases.

b) As pressure increases burning rate increases.

c) As initial temperature increases burning rate increases.

d) As initial temperature increases the pressure index or exponent of Sant Robert relation decreases.

e) Changing particle size distribution is more practical than changing initial temperature, which is usually the ambient or surrounding atmosphere temperature. It is also more practical than changing pressure which is predetermined according to requirements of rocket motor mechanical design and required ballistics characteristics 


\section{References}

[1] SRM Manual Design, Adjustment of Burning Rate of Propellant, 2003.

[2] Baily, A., and Murray S. G., Explosives, Propellants and Pyrotechnics, 1'st edition, John Wiley \& Sons, USA 1989.

[3] http://en.Wikipedia.org, Wikipedia Encyclopedia, Le Chatelier's principle,6 p.m. 25 Jan. 2011.

[4] Brill, T. B. and Budenz B. T., Flash Pyrolysis of Ammonium Perchlorate Hydroxyl Terminated Poly Butadiene Mixtures Including Selected Additives, American Institute of Aeronautics and Astronautics, Inc., Virginia, 2000.

[5] Smith, J. M. and Van Ness, H. C., Introduction to Chemical Engineering Thermodynamics, 4th ed., Mc Graw-Hill, 1987.

[6] Sutton G. P., Rocket propulsion Elements, 6th Edition, John Wiley \& Sons Inc, USA, 1992.

[7] Fedoroff, B. T., Encyclopedia of Explosives and Related Items Vol. 2-2, Picatinny Arsenal, USA, 1960.

[8] Voultine, S., Rocket propulsion materials, Military Engineering College, Iraq, 1992.

Table 1 Propellant Ingredients and their Functions.

\begin{tabular}{|c|c|c|}
\hline No & Name & Function \\
\hline 1 & $\begin{array}{l}\text { AP fine } \quad[5-10 \mu \mathrm{m}] \\
\text { AP medium }[60-80 \mu \mathrm{m}] \\
\text { AP coarse }[100-140 \mu \mathrm{m}]\end{array}$ & Oxidizer \\
\hline 2 & HTPB & Pre-polymer and Fuel Source \\
\hline 3 & $\mathrm{Al}$ & Metal Fuel \\
\hline 4 & MAPO & Bonding Agent \\
\hline 5 & TBFe & Burning Rate Accelerator \\
\hline 6 & TDI & Curing Agent ( cross linker) \\
\hline 7 & DOS & Plasticizer \\
\hline 8 & Styrene $(\mathrm{Bx})$ & Processing Agent \\
\hline 9 & N-N diphenyl-P-phenylene diamine $(\mathrm{H})$ & Aging Agent \\
\hline 10 & Cesium oxide $(\mathrm{X}-858)$ & Antioxidant \\
\hline 11 & HX-103 & Antioxidant \\
\hline
\end{tabular}

Table 2 Results of Strand Burner Tests

\begin{tabular}{|c|c|c|c|c|c|c|}
\hline \multirow{2}{*}{ Formulation } & \multirow{2}{*}{$\begin{array}{c}\text { Temperature } \\
\left({ }^{\circ} \mathrm{C}\right)\end{array}$} & & \multicolumn{4}{|c|}{ Pressure $(\mathrm{MPa})$} \\
\hline & & & 4 & 6 & 9 & 11 \\
\hline F1 & 10 & \multirow{9}{*}{ 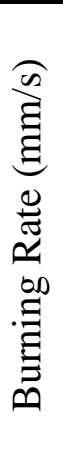 } & 9.8260 & 12.841 & 13.187 & 14.170 \\
\hline F1 & 20 & & 10.608 & 12.605 & 13.867 & 14.294 \\
\hline $\mathrm{F} 1$ & 30 & & 10.680 & 12.369 & 15.023 & 15.789 \\
\hline$\overline{\mathrm{F} 2}$ & 10 & & 13.298 & 15.048 & $17 \overline{17.881}$ & 19.447 \\
\hline $\mathrm{F} 2$ & 20 & & 13.430 & 15.249 & 18.422 & 20.278 \\
\hline $\mathrm{F} 2$ & 30 & & 13.526 & 15.801 & 19.338 & 20.795 \\
\hline F3 & 10 & & 8.995 & 9.917 & 11.377 & 12.615 \\
\hline $\mathrm{F} 3$ & 20 & & 9.555 & 10.368 & 11.762 & 12.849 \\
\hline F3 & 30 & & 9.62 & 11.388 & 12.471 & 13.314 \\
\hline
\end{tabular}


Table 3 Results of BEM Tests at different AP Particle Size Distributions.

\begin{tabular}{c|c|c}
\hline \hline Formulation & $\begin{array}{c}\text { Average Pressure } \\
(\mathrm{MPa})\end{array}$ & $\begin{array}{c}\text { Burning Rate } \\
(\mathrm{mm} / \mathrm{s})\end{array}$ \\
\hline F1 & 4.146 & 14.127 \\
\hline F2 & 6.474 & 17.346 \\
\hline F3 & 3.088 & 9.05 \\
\hline
\end{tabular}

Table 4 Values of Temperature Coefficient (a) and Exponent (n) at Tests Temperatures and Particles Size Distributions

\begin{tabular}{c|c|c|c}
\hline Formulation & Temperature $\left({ }^{\circ} \mathrm{C}\right)$ & $\mathrm{a}$ & $\mathrm{n}$ \\
\hline F1 & 10 & 6.119 & 0.400 \\
\hline F1 & 20 & 6.819 & 0.313 \\
\hline F1 & 30 & 6.918 & 0.303 \\
\hline \hline F2 & 10 & 7.332 & 0.436 \\
\hline F2 & 20 & 7.371 & 0.419 \\
\hline F2 & 30 & 7.691 & 0.385 \\
\hline \hline F3 & 10 & 5.524 & 0.305 \\
\hline F3 & 20 & 6.219 & 0.296 \\
\hline F3 & 30 & 6.428 & 0.337 \\
\hline \hline
\end{tabular}

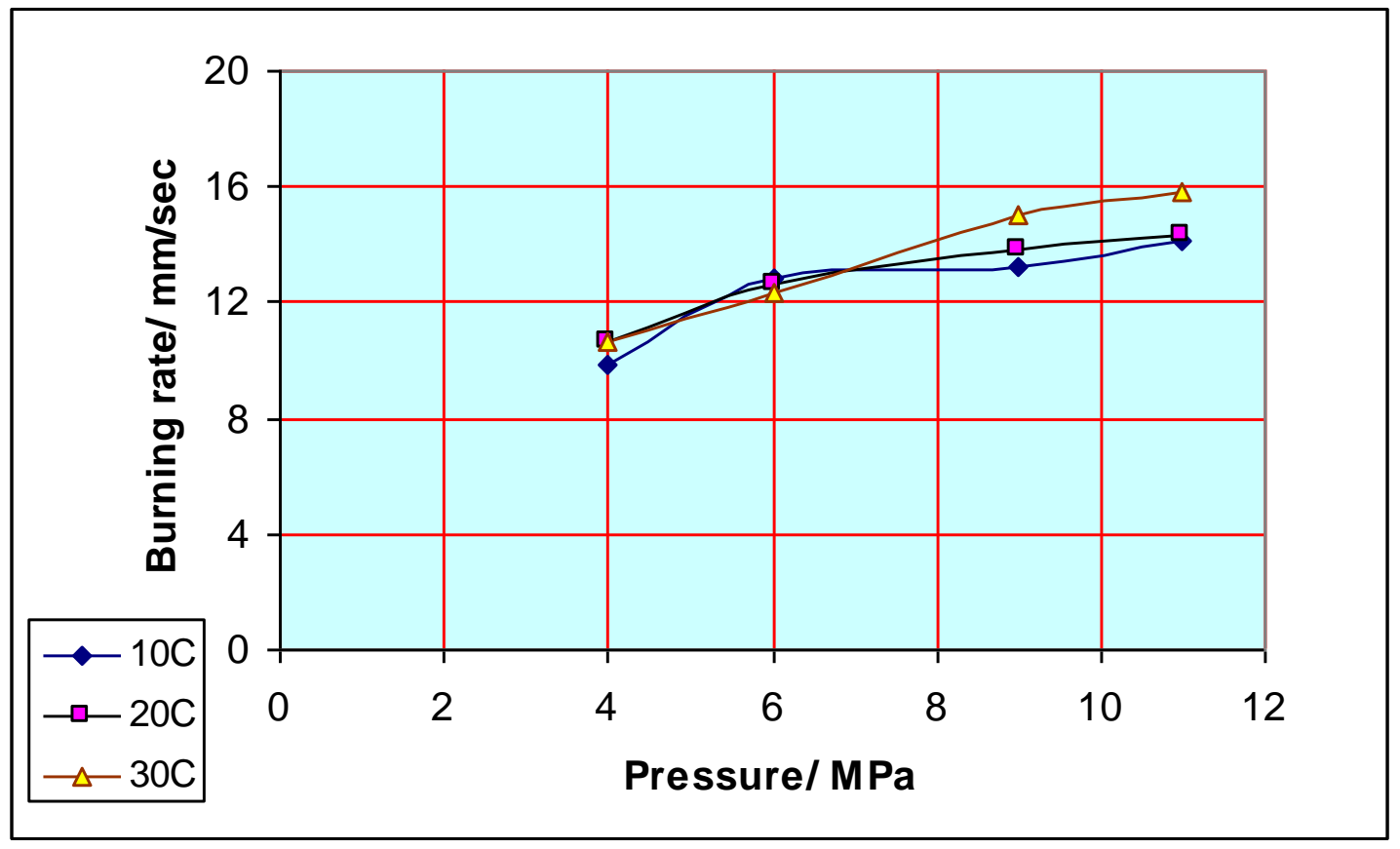

Fig. 1 Burning Rate - Pressure Relation for Formulation F1 


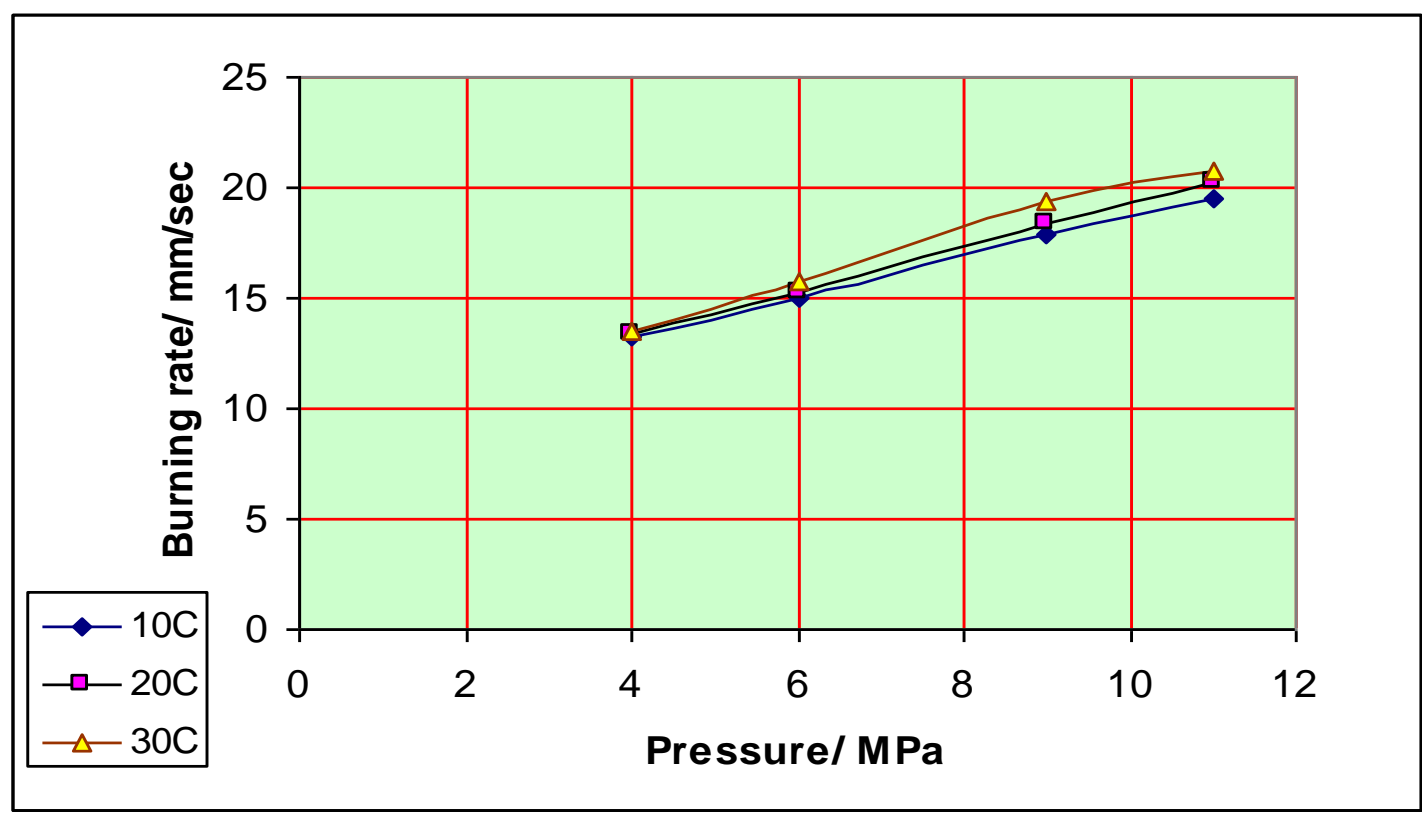

Fig. 2 Burning Rate - Pressure Relation for Formulation F2

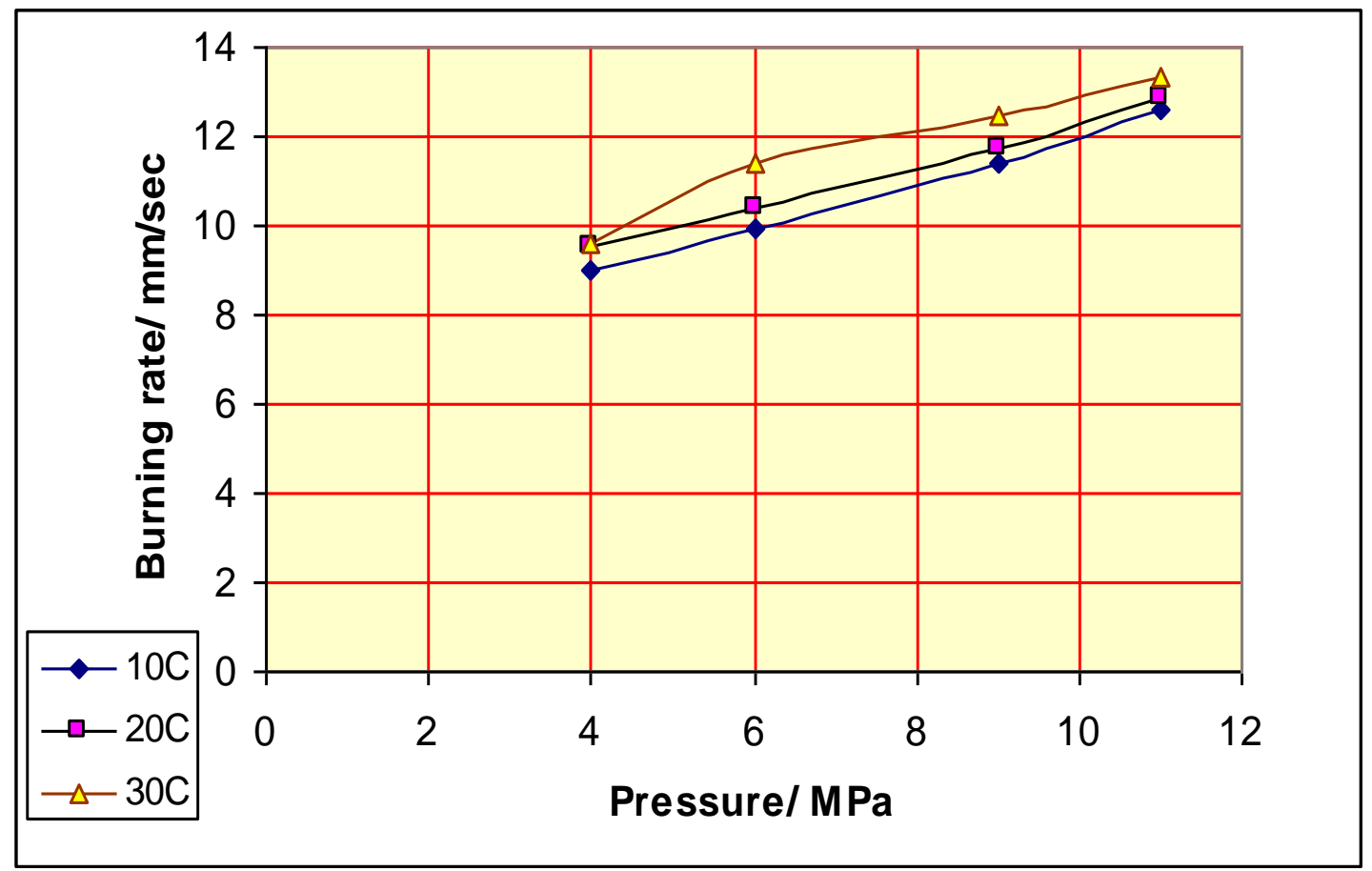

Fig. 3 Burning Rate - Pressure Relation for Formulation F3 